\title{
Validation of Tools to Accelerate High-Speed CFD Simulations Using OpenFOAM
}

\author{
Daniel E. R. Espinoza, Thomas J. Scanlon ${ }^{\dagger}$ \\ and Richard E. Brown \\ Centre for Future Air-Space Transportation Technology, \\ University of Strathclyde, Glasgow, G1 1XJ, United Kingdom
}

Local time stepping (LTS) and adaptive mesh refinement (AMR) have been implemented into rhoCentralFoam, a compressible solver within the open source computational fluid dynamics (CFD) code OpenFOAM. The LTS solver has been validated using a compressible Couette channel with heat transfer and a supersonic flat plate. An excellent concurrence is found for these cases in comparison with the solutions with the obtained results being, respectively, 2.56 and 8.96 times faster compared to the unmodified solver. The AMR solver was employed to simulate hypersonic flow over a $30^{\circ}$ wedge, and the Sod shock tube test case, and has also achieved satisfactory agreement with the analytical solutions. These results highlight the potentially significant computational cost savings that may be achieved when solving high-speed, compressible flows using this approach.

\section{Nomenclature}

F $\quad$ Flux array

S Source terms array

W Conserved variables array

$\vec{v} \quad$ Velocity vector, $\mathrm{m} / \mathrm{s}$

a Acceleration factor

c Courant number

$H \quad$ Height of the channel, $\mathrm{m}$

$k \quad$ Thermal conductivity, $\mathrm{W} /(\mathrm{m} \cdot \mathrm{K})$

$L \quad$ Length of the shock tube

$M \quad$ Mach number

$p \quad$ Pressure, $\mathrm{Pa}$

Re Reynolds number

$T$ Temperature, $\mathrm{K}$

$t \quad$ Time, s

$U \quad$ Flow speed in the $\mathrm{x}$ direction, $\mathrm{m} / \mathrm{s}$

$x \quad$ Horizontal coordinate, $\mathrm{m}$

$y \quad$ Vertical coordinate, $\mathrm{m}$

AMR Adaptive Mesh Refinement

CFD Computational Fluid Dynamics

DPLR Data-Parallel Line Relaxation
LAURA Langley Aerothermodynamic Upwind Relaxation Algorithm

LTS Local time stepping

OpenFOAM Open Source Field Operation And Manipulation

TVD Total Variation Diminishing

$\begin{array}{ll}\text { Subscripts } \\ \infty & \text { Upstream conditions } \\ B W & \text { Bottom wall } \\ l & \text { Initial conditions at left half of shock tube } \\ \max & \text { Maximum } \\ r & \text { Initial conditions at right half of shock tube } \\ T W & \text { Top wall }\end{array}$

Symbols

$\Delta t \quad$ Time-step, s

$\mu \quad$ Dynamic viscosity, $\mathrm{Pa} \cdot \mathrm{s}$

$\nabla \quad$ Gradient operator

$\rho \quad$ Density, $\mathrm{kg} / \mathrm{m}^{3}$

\footnotetext{
*Ph.D. Student, Department of Mechanical and Aerospace Engineering, AIAA student member

${ }^{\dagger}$ Senior Lecturer, Department of Mechanical and Aerospace Engineering

$¥$ Professor, Department of Mechanical and Aerospace Engineering, AIAA Senior member
} 


\section{Introduction}

To be able to satisfactorily capture the essential flow features in the simulation of hypersonic flows, good practice dictates that a specific set of procedures should be followed. These include the categorisation of the correct physics (e.g. non-equilibrium effects, ${ }^{1}$ modelling of turbulence ${ }^{2}$ ) as well as the selection of the most appropriate solution methodologies to capture the essential physics (e.g. shock-capturing and shock-fitting approaches, ${ }^{3}$ choice of TVD schemes ${ }^{4,5}$ ). Whereas a significant amount of research has been dedicated to these areas, this paper focuses on how the spatial and temporal resolution of calculations may be improved in order to achieve satisfactory numerical results with an accompanying reduction in computational expense.

With regard to spatial resolution, a computational mesh that attempts to follow the shock structure is an essential feature of the solution methodology. In terms of the cell shape, a tetrahedral mesh can be created more quickly than a structured one and delivers greater flexibility, in particular for complex geometries. Poor predictions of heat transfer can result ${ }^{6}$ however, and, for this reason, standard practice for unstructured meshes is to attempt to have prismatic cells aligned with solid surfaces and the shock wave itself. $^{7}$ This alignment of the grid with the shock wave is particularly important in order to avoid significant fluctuations occurring in the prediction of the stagnation point heat flux. ${ }^{8}$ In addition, a sufficient degree of cell refinement is required near the shock wave simply in order to properly capture the spatial variation in the flow properties there. ${ }^{9}$ In this paper we present a mesh refinement strategy which significantly improves the capturing of spatial gradients in the flow field.

The rate of temporal advance of the computation must also be such that the numerical stability and accuracy of the calculation are preserved. We present a time-acceleration technique which shows potential to greatly accelerate the computation of the initial transient within the computation and hence the rate of convergence of the calculation towards its steady state.

Some well-known hypersonic codes (e.g. LAURA ${ }^{10}$ and DPLR ${ }^{11}$ ) contain features to deal with the spatial and temporal issues described above. Although the open source computational fluid dynamics code used in this paper, OpenFOAM, ${ }^{12}$ does have such capabilities, the libraries that include these features have yet to be included in its high-speed compressible solvers. The objective of this paper is to incorporate local time stepping (LTS) and adaptive mesh refinement (AMR) into the OpenFOAM high-speed compressible solver rhoCentralFoam, and to validate the results of computations using this new approach against appropriate analytical solutions or experimental data.

\section{Methodology}

OpenFOAM is an open source computational fluid dynamics (CFD) software package consisting of a set of flexible $\mathrm{C}++$ modules to resolve complex fluid flows. The package includes tools for meshing and preand post-processing, as well as solvers for both incompressible and compressible flows. ${ }^{12}$

Amongst the compressible solvers that are available in OpenFOAM, rhoCentralFoam ${ }^{13}$ has been chosen for the simulations presented in this work, largely because of its satisfactory performance in previouslyconducted high-speed compressible flow simulations. ${ }^{13,14}$ rhoCentralFoam is an unsteady, compressible solver, that uses semi-discrete, non-staggered, Godunov-type central ${ }^{15}$ and upwind-central ${ }^{16}$ schemes. These schemes both avoid the explicit need for a Riemann solver, resulting in a numerical approach that is both simple and efficient. ${ }^{16}$

\section{II.A. Local time stepping}

Local time stepping (LTS) is a technique used to accelerate the convergence of computations towards their steady-state solutions. As rhoCentralFoam is an unsteady solver, the solution goes through an initial transient before reaching steady state. In the present form of rhoCentralFoam, the rate of temporal advance is the same for all the cells in the computational domain, and is thus limited by the smallest time-step 
allowed for any cell. LTS allows each cell to advance in time at their own time-step.

While the Navier-Stokes equations have the form

$$
\frac{\partial \mathbf{W}}{\partial t}+\nabla \cdot \mathbf{F}=\mathbf{S}
$$

where $\mathbf{W}$ represents the conserved variables, $\mathbf{F}$ the fluxes, $\mathbf{S}$ the source terms, $t$ the time, and $\nabla$ the gradient operator, the LTS technique solves the equation

$$
\frac{1}{a} \frac{\partial \mathbf{W}}{\partial t}+\nabla \cdot \mathbf{F}=\mathbf{S}
$$

where $a$ is an acceleration factor that depends on $\mathbf{W}$.

If the solution is steady, then the time derivative is exactly zero, and so a solution to Equation (2) is also a solution of Equation (1), independent of the value of $a$.

In the LTS procedure that has been implemented into OpenFOAM, the time derivative is modelled using an Euler scheme. Based on similar implementations in the OpenFOAM solver LTSInterFoam and those found in Ref. 17, the time-step $\Delta t$ for each cell is selected individually as the minimum between the global, user-defined maximum time-step $\Delta t_{\max }$, and the time-step that is determined by the maximum allowable Courant number $c_{\max }$ within the flow. In other words

$$
\Delta t=\min \left(\Delta t_{\max }, \Delta t\left(c_{\max }\right)\right)
$$

In practice, the field $\Delta t$ calculated using this approach is first smoothed spatially before being applied to the calculation of the LTS solution in each computational cell.

\section{II.B. Adaptive Mesh Refinement}

Adaptive mesh refinement (AMR) is a technique that is used to modify an existing mesh so as to improve its resolution of the flow properties in regions of interest, and thus to capture the flow with enhanced accuracy without an excessive increase in computational effort. The advantage of implementing this technique within OpenFOAM is that it allows the details of the flow near shocks to be captured without the need for an excessively fine mesh throughout the entire computational domain. Based on the implementations within the OpenFOAM solvers interDyMFoam and rhoCentralDyMFoam, the computational mesh is refined or coarsened locally during the computation in order to adapt to the spatial gradients within the flow properties. The approach uses a tree structure, and the maximum coarsening that can be obtained is equivalent to the cell density of the base mesh. When refining the mesh, the algorithm splits each hexahedral cell in two along each coordinate direction to generate eight new cells from the original parent cell. Figure 1 shows the refinement algorithm that is used by the AMR procedure and Figure 2 illustrates an example of a parent-child tree that could be obtained when a cell is split using this procedure.

\section{II.C. Test cases}

The following test cases have been used to validate the LTS and AMR algorithms as implemented within rhoCentralFoam.

\section{II.C.1. LTS}

For the purposes of validating the LTS procedure, a Couette flow and a supersonic flat plate have been simulated.

For the Couette flow, the separation between the upper and lower walls was $1 \mathrm{~m}$, and the lateral boundaries of the computation, modelled using cyclic boundary conditions, were placed $40 \mathrm{~cm}$ apart. The computational 


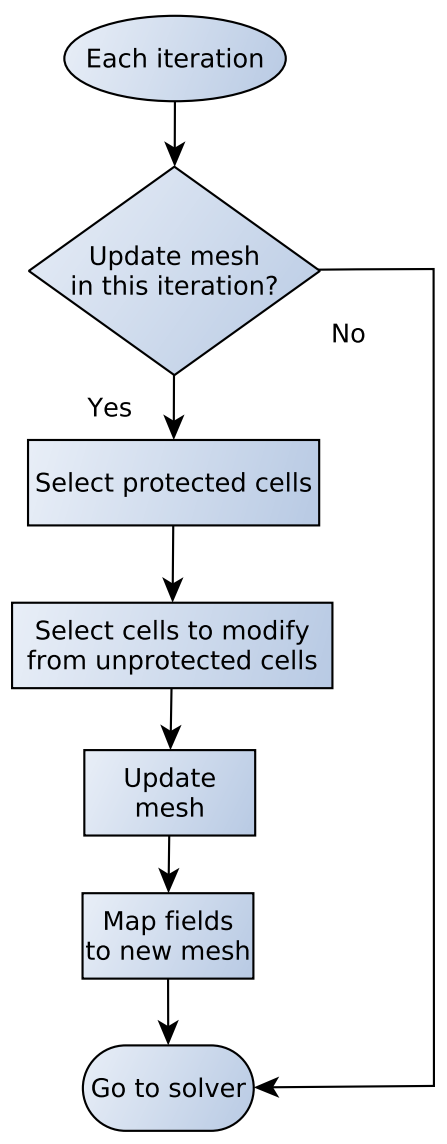

Figure 1. Flow chart for the refinement algorithm

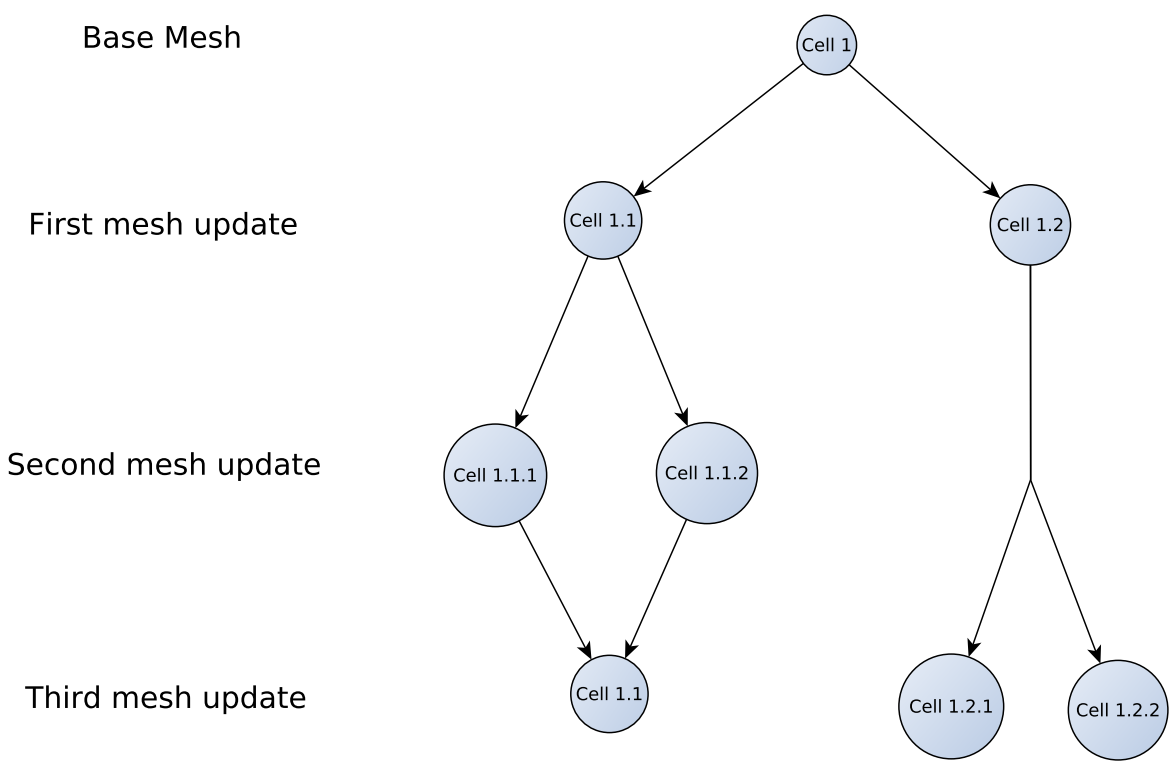

Figure 2. Parent-child tree for cell refinement and coarsening 


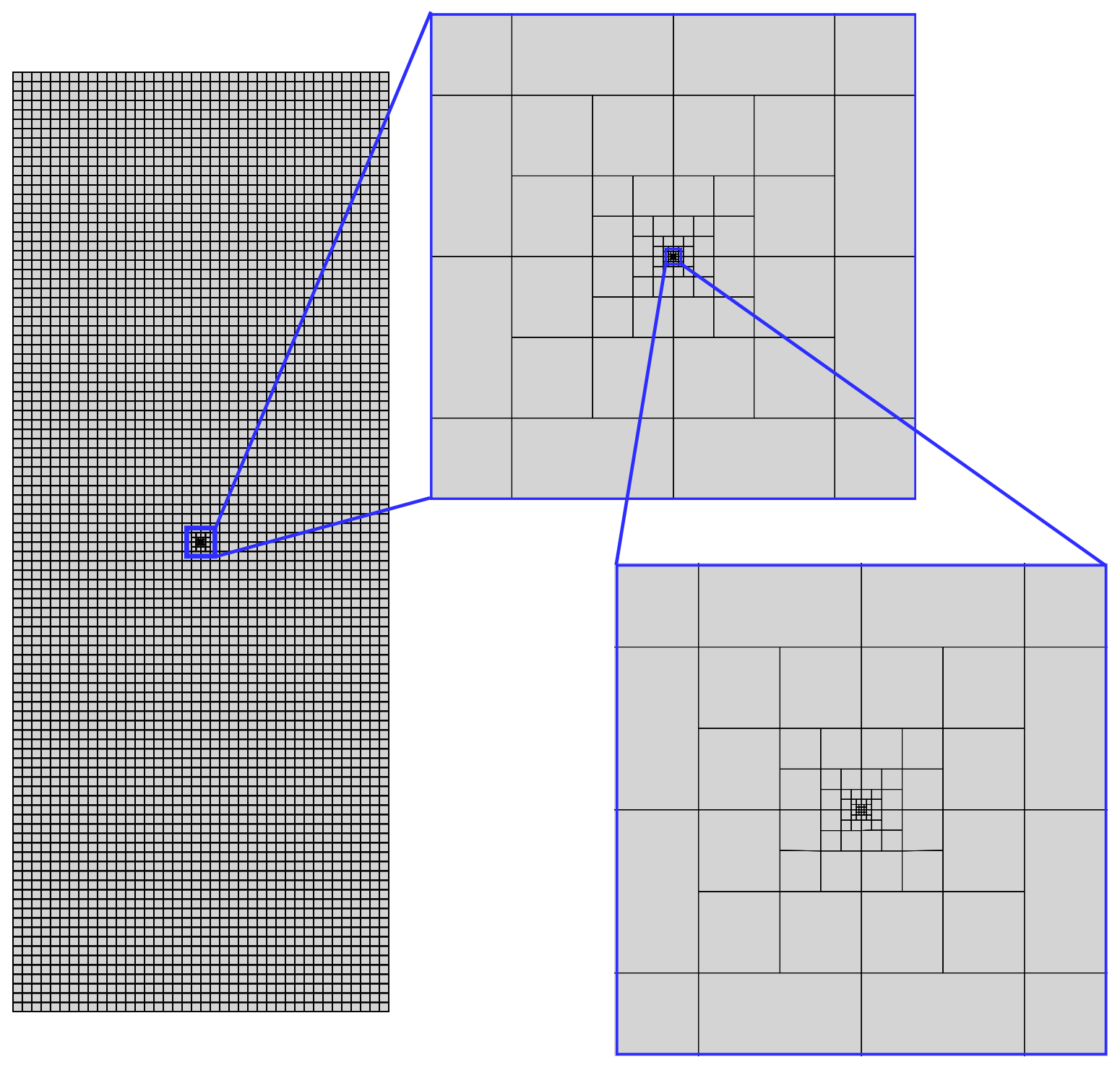

Figure 3. Mesh for the Couette flow test case 
domain is illustrated in Figure 3. The base cell size was set to $0.01 \mathrm{~m} \times 0.01 \mathrm{~m}$. Four cells were refined at the centre of the channel, then the four cells at the centre of the group of refined cells, and so on until 11 levels of refinement were achieved. The finest cell has a size of $\approx 4.88 \mu \mathrm{m} \times 4.88 \mu \mathrm{m}$, as can be seen in Figure 3 .

The fluid within the channel is air, and the initial and boundary conditions are set to

$$
\begin{aligned}
& \vec{v}(t=0)=0 \mathrm{~m} / \mathrm{s} \\
& T(t=0)=200 K \\
& p(t=0)=100 P a \\
& U_{T W}=200 \mathrm{~m} / \mathrm{s} \\
& T_{T W}=400 K \\
& U_{B W}=0 \mathrm{~m} / \mathrm{s} \\
& T_{B W}=200 K
\end{aligned}
$$

where $\vec{v}$ represents the velocity vector, $T$ the temperature, $p$ the pressure, $U$ the flow speed in the $x$-direction, and the subscripts $T W$ and $B W$ refer to the top and bottom walls of the channel respectively.

The maximum allowable courant number in the flow was set to 0.2 and the maximum allowable time-step to $1 \times 10^{-3} \mathrm{~s}$.

For the supersonic flat plate, ${ }^{18}$ the length of the plate within the domain was $0.1 \mathrm{~m}$, with the domain extending $5 \times 10^{-3} \mathrm{~m}$ behind the plate. Figure 4 shows the 2-Dimensional computational domain, with the flate plate in red, and consecutive zoom to be able to appreciate the mesh in detail. The smallest cells, at the leading edge of the plate, have a size of $1 \mu \mathrm{m} \times 1 \mu \mathrm{m}$, whereas the coarsest cell, at the top right corner, has a size of $\approx 2.8 \mathrm{~mm} \times 2.8 \mathrm{~mm}$.

The fluid within the domain is air, and the initial and boundary conditions are set so to match the Reynolds $(R e)$ and Mach $(M)$ numbers to those is Ref. 18, as well as the flat plate/upstream temperature ratio (that is, $R e=500, M_{\infty}=2$ and $\frac{T_{W}}{T_{\infty}}=2$ ). Setting the upstream temperature to $300 \mathrm{~K}$, and using $0.01 \mathrm{~m}$ as the reference length to calculate the Reynolds number, the initial and boundary conditions result in:

$$
\begin{aligned}
& U(t=0)=694.55 \mathrm{~m} / \mathrm{s} \\
& T(t=0)=300 K \\
& T_{\infty}=300 K \\
& \begin{array}{r}
p(t=0)=114.46 P a \\
p_{\infty}=114.46 P a
\end{array}
\end{aligned}
$$

where the subscript $\infty$ refers to upstream conditions. The flat plate temperature is set to $600 \mathrm{~K}$ and no-slip wall boundaries are assumed. The maximum allowable courant number in the flow was set to 0.1 and the maximum allowable time-step to $1 \times 10^{-5} \mathrm{~s}$. 


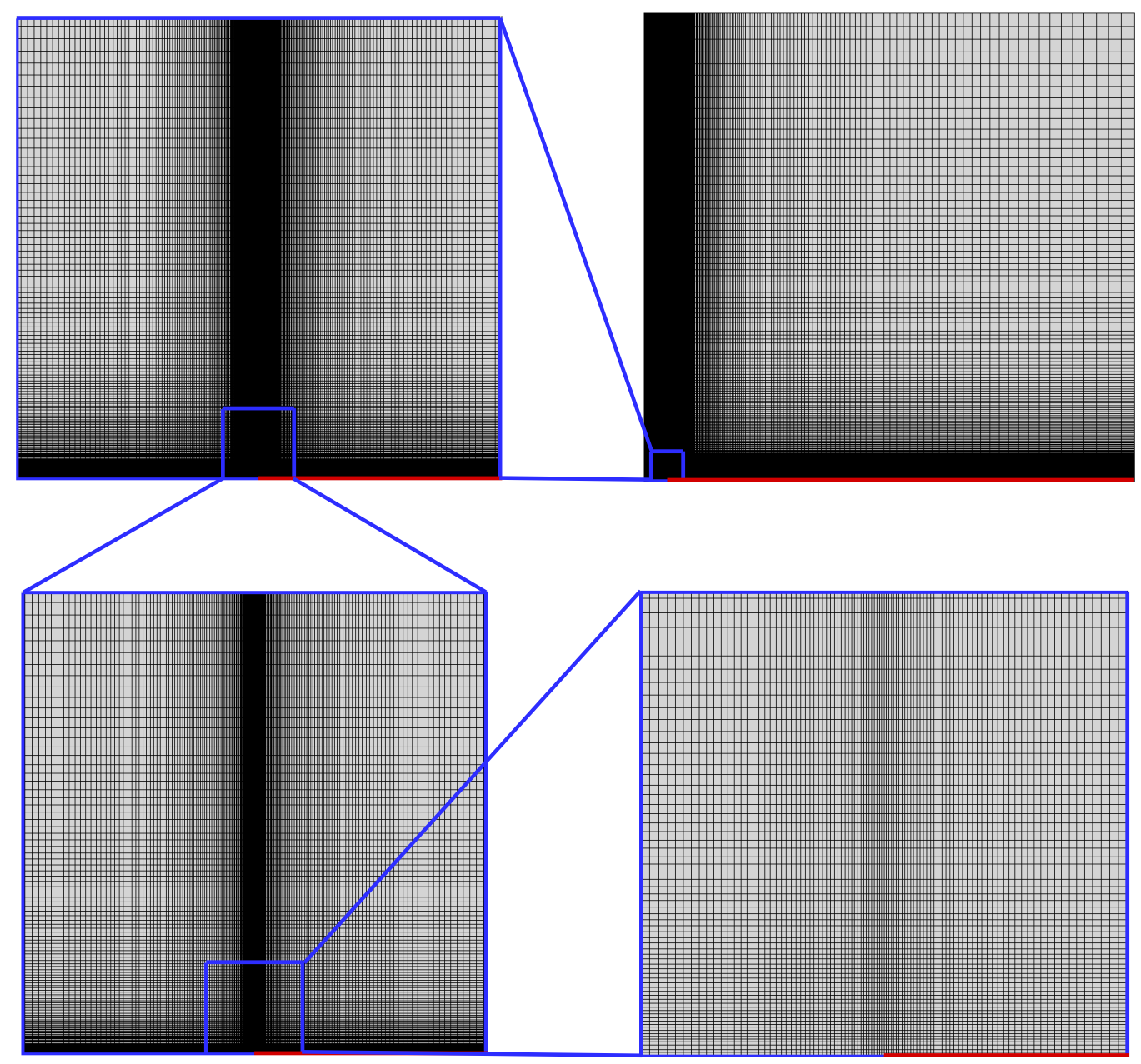

Figure 4. Mesh for the flat plate test case 


\section{II.C.2. $A M R$}

A first verification of the implementation of the AMR technique within rhoCentralFoam was conducted by modelling the inviscid flow at Mach 5 over a two-dimensional $30^{\circ}$ wedge. The initial mesh can be seen in Figure 5 .

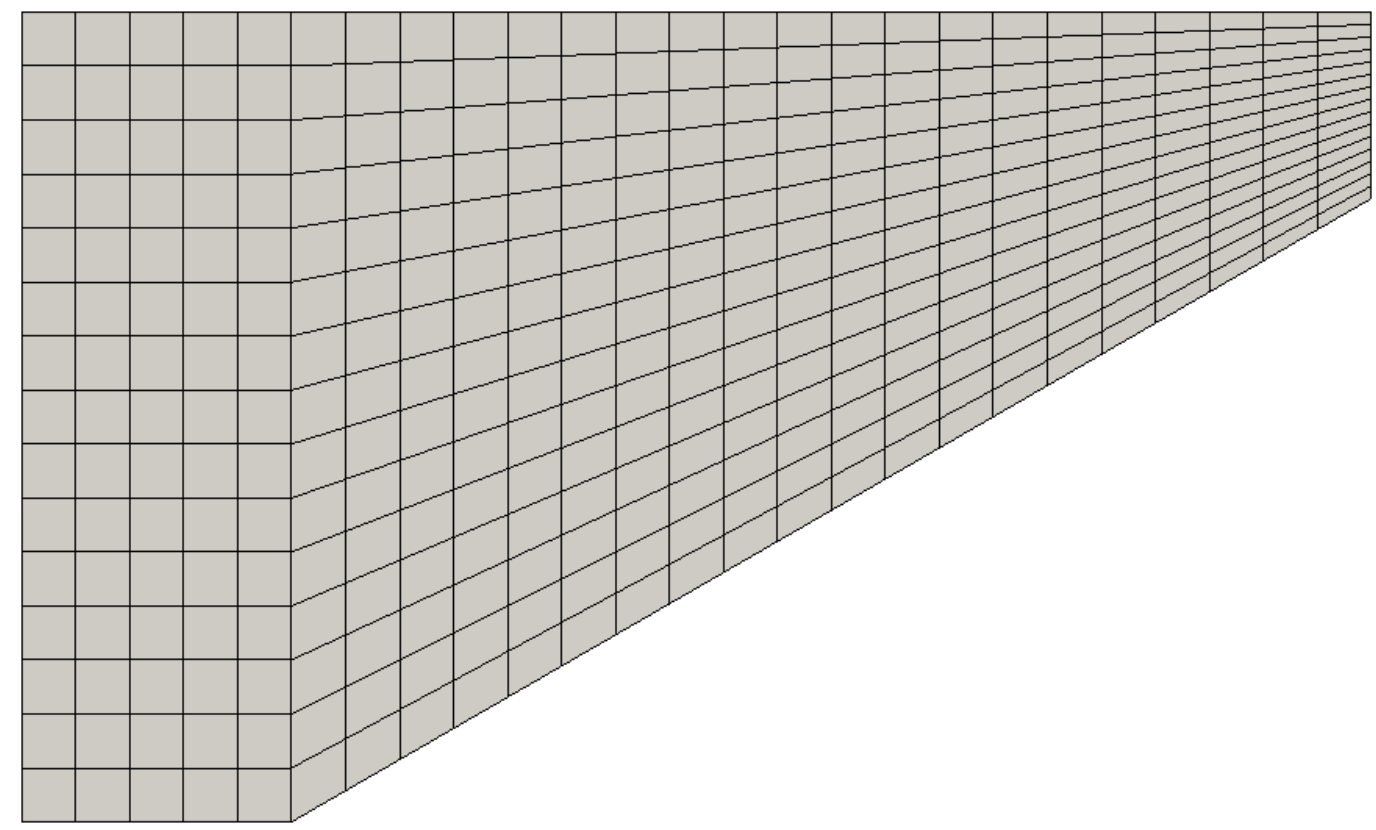

Figure 5. Initial mesh for the supersonic wedge test case

The fluid used for this simulation has been set up so to resolve the non-dimensional problem. As such, the initial and boundary conditions are

$$
\begin{aligned}
\vec{v}(t=0) & =0 \\
T(t=0) & =1 \\
p(t=0) & =1
\end{aligned}
$$

$$
\begin{aligned}
M_{\infty} & =5 \\
T_{\infty} & =1 \\
p_{\infty} & =1
\end{aligned}
$$

No-slip, adiabatic wall boundaries are assumed.

Mesh refinement was instigated when $\|\nabla M\|>1$, and coarsening when $\|\nabla M\|<1$. A million time-steps were executed between each mesh update.

In addition, the Sod shock tube test case ${ }^{19}$ was used to study the suitability of the AMR technique for transient simulations. The initial mesh can be seen in Figure 6 .

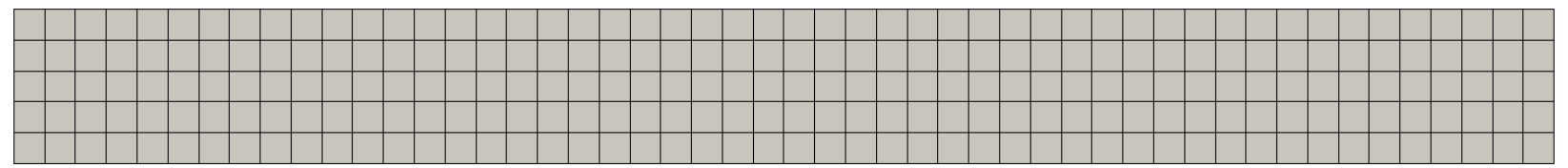

Figure 6. Initial mesh for the shock tube test case

The fluid used for this simulation has been set up so to resolve the non-dimensional problem. As such, 
the initial conditions are

$$
\begin{array}{ll}
u(x / L<0, t=0)=u_{l}=0 & u(x / L>0, t=0)=u_{r}=0 \\
p(x / L<0, t=0)=p_{l}=1 & p(x / L>0, t=0)=p_{r}=0.1 \\
\rho(x / L<0, t=0)=\rho_{l}=1 & \rho(x / L>0, t=0)=\rho_{r}=0.125
\end{array}
$$

where $L$ represents the length of the shock tube, $\rho$ the density of the fluid, and the subscripts $l$ and $r$ refer to the left and right half of the shock tube respectively.

Two different refinement/coarsening criteria were compared. For the first criterion, mesh refinement was instigated when $\left\|\frac{\nabla p}{p}\right\|>1$, and coarsening when $\left\|\frac{\nabla p}{p}\right\|<0.5$. For the second, the mesh was refined when $\left\|\frac{\nabla \rho}{\rho}\right\|>1$ and coarsened when $\left\|\frac{\nabla \rho}{\rho}\right\|<0.5$. For both cases, five time-steps were executed between each mesh update.

\section{Results and discussion}

\section{III.A. LTS}

\section{III.A.1. Couette flow}

The figures presented below show the flow properties along the vertical centreline through the Couette channel and are compared with the analytical solution for the flow, namely:

$$
\begin{aligned}
T(y) & =T_{B W}+\left(T_{T W}-T_{B W}\right) \frac{y}{H}+\frac{\mu U_{T W}^{2}}{2 k} \frac{y}{H}\left(1-\frac{y}{H}\right) \\
U(y) & =U_{T W} \frac{y}{H} \\
V(y) & =0 \mathrm{~m} / \mathrm{s} \\
p(y) & =\mathrm{constant}
\end{aligned}
$$

where $H$ is the height of the channel, $y$ the vertical coordinate, $\mu$ the dynamic viscosity of the flow, and $k$ its thermal conductivity.

Figures 7 and 8 show the progress of the solution with respect to the number of iterations. Similar results were found for pressure and $y$-velocity. It is evident that the LTS solver converges to the analytical solution using much fewer iterations than the unmodified solver. Table 1 shows the individual cell time-step. Whereas the time-step for the unmodified solver is the same for all cells, for the LTS solver the minimum time-step is two orders of magnitude smaller than the maximum time-step. This difference allows the bigger cells, which have larger time-steps, to reach a steady state solution faster, accelerating the global convergence of the LTS solution.

Using the LTS solver, after 7.5 million iterations the maximum relative error is approximately $1 \%$. However, for the unmodified rhoCentralFoam even after 27.5 million iterations the maximum relative error is still greater than that achieved by the LTS method in 7.5 million iterations. The computational time required to reach these numbers of iterations can be seen in Table 2. The LTS solver employed 2.56 times less computational time than the unmodified solver to reach a relative error of approximately $1 \%$. 


\begin{tabular}{|c|c|c|c|c|c|c|c|c|c|}
\hline It $2.5 \mathrm{M}$ & - & It $7.5 \mathrm{M}$ & - & It $12.5 \mathrm{M}$ & -- & It $17.5 \mathrm{M}$ & & It $22.5 \mathrm{M}$ & $--\quad$ It 27. \\
\hline It & - & It $10.0 \mathrm{M}$ & 一 & It $15.0 \mathrm{M}$ & -- & It $20.0 \mathrm{M}$ & -- & It $25.0 \mathrm{M}$ & Analytical \\
\hline
\end{tabular}

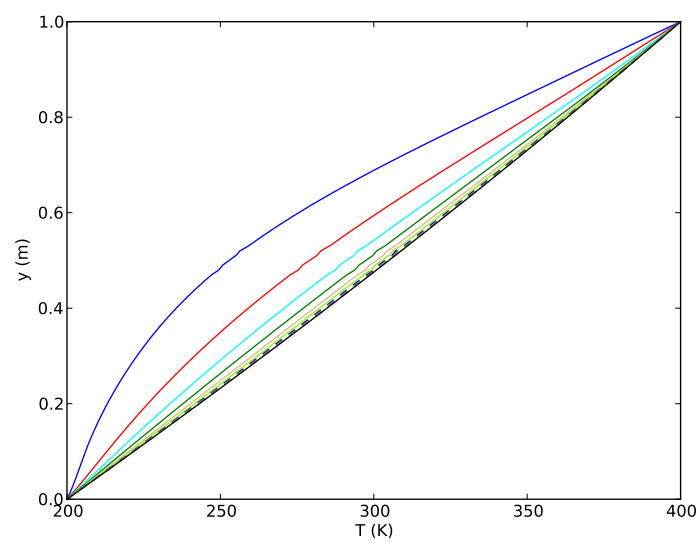

(a) Unmodified solver: $\mathrm{T}$

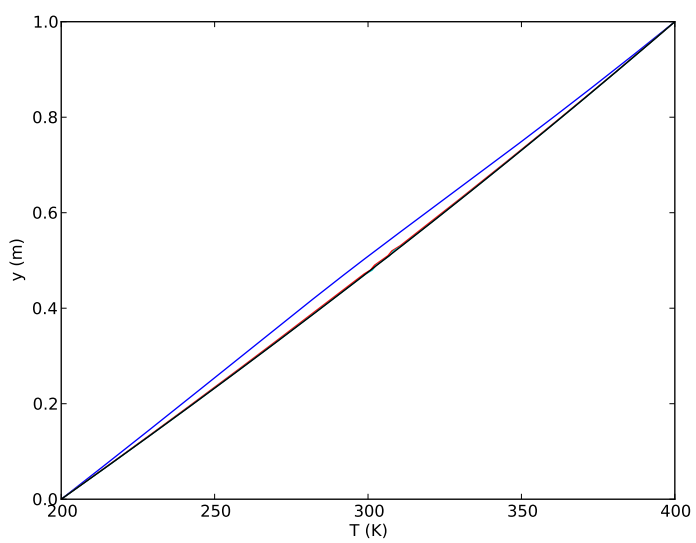

(b) LTS solver: T

Figure 7. Results from the unmodified and the LTS solvers for temperature

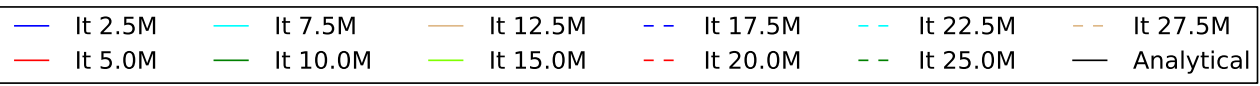

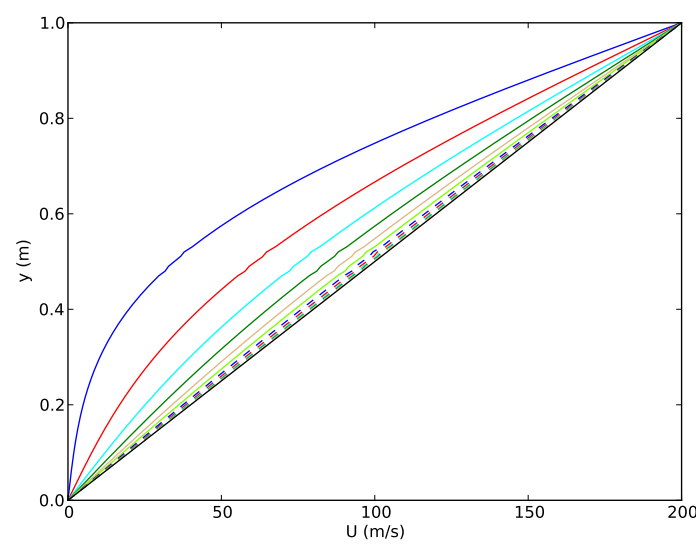

(a) Unmodified solver: U

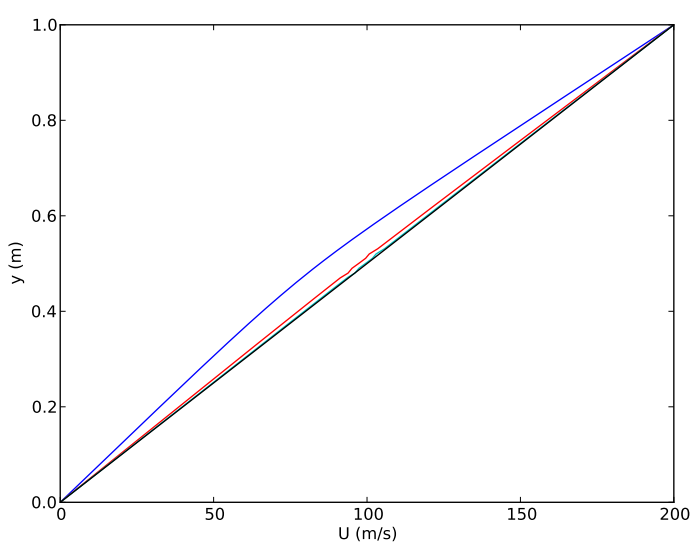

(b) LTS solver: U

Figure 8. Results from the unmodified and the LTS solvers for $x$-velocity 


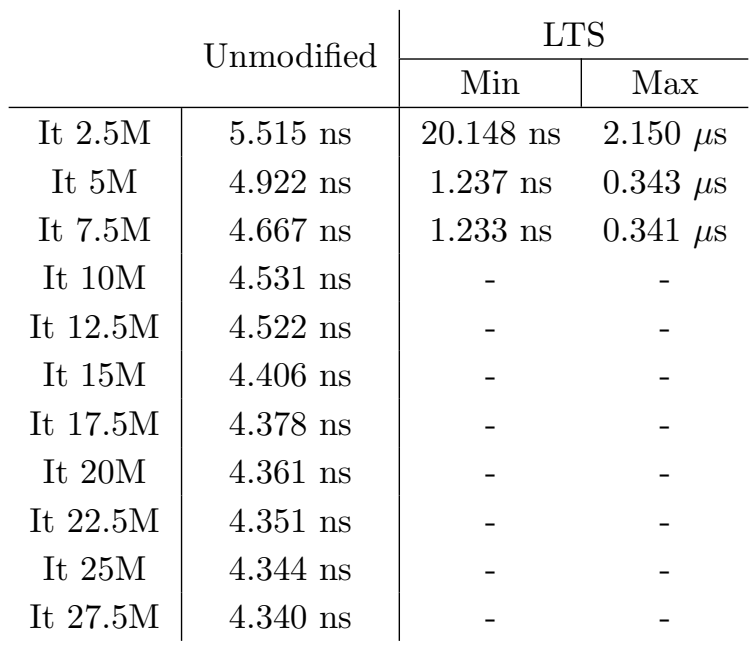

Table 1. Simulation time-step in each iteration level, for the unmodified and LTS solvers

\begin{tabular}{|c|c|c|}
\hline & Unmodified & LTS \\
\hline It $2.5 \mathrm{M}$ & $7 \mathrm{~h} 26 \mathrm{~min} 50 \mathrm{~s}$ & $9 \mathrm{~h} 21 \mathrm{~min} 17 \mathrm{~s}$ \\
\hline It $5 \mathrm{M}$ & $14 \mathrm{~h} 55 \mathrm{~min} 57 \mathrm{~s}$ & $18 \mathrm{~h} 52 \mathrm{~min} 40 \mathrm{~s}$ \\
\hline It $7.5 \mathrm{M}$ & $22 \mathrm{~h} 37 \mathrm{~min} 11 \mathrm{~s}$ & $28 \mathrm{~h} 0 \mathrm{~min} 44 \mathrm{~s}$ \\
\hline It $10 \mathrm{M}$ & $29 \mathrm{~h} 23 \mathrm{~min} 21 \mathrm{~s}$ & - \\
\hline It $12.5 \mathrm{M}$ & $35 \mathrm{~h} 5 \mathrm{~min} 21 \mathrm{~s}$ & - \\
\hline It $15 \mathrm{M}$ & $41 \mathrm{~h} 13 \mathrm{~min} 13 \mathrm{~s}$ & - \\
\hline It $17.5 \mathrm{M}$ & $47 \mathrm{~h} 12 \mathrm{~min} 38 \mathrm{~s}$ & - \\
\hline It $20 \mathrm{M}$ & $52 \mathrm{~h} 58 \mathrm{~min} 3 \mathrm{~s}$ & - \\
\hline It $22.5 \mathrm{M}$ & $59 \mathrm{~h} 21 \mathrm{~min} 11 \mathrm{~s}$ & - \\
\hline It $25 \mathrm{M}$ & $65 \mathrm{~h} 30 \mathrm{~min} 11 \mathrm{~s}$ & - \\
\hline It $27.5 \mathrm{M}$ & $71 \mathrm{~h} 41 \mathrm{~min} 41 \mathrm{~s}$ & - \\
\hline
\end{tabular}

Table 2. Computational time required to reach each iteration level, for the unmodified and LTS solvers 


\section{III.A.2. Supersonic flat plate}

The following data show the flow properties along the vertical line located one reference length $(0.01 \mathrm{~m})$ behind the leading edge of the flat plate.

The simulation has been considered converged when the difference between two solutions $5 \times 10^{4}$ iterations apart was smaller than $0.01 \%$ of the upstream values. The unmodified solver reached $1.7 \times 10^{6}$ iterations before this condition was fulfilled, whereas for the LTS solver only $1.5 \times 10^{5}$ iterations were required. The computational times required to reach this convergence criterion were $86 \mathrm{~h} 48 \mathrm{~min} 56 \mathrm{~s}$ for the unmodified solver and $9 \mathrm{~h} 41 \mathrm{~min} 21 \mathrm{~s}$ for LTS solver, thus achieving convergence 8.96 faster than the unmodified solver.

Figures 9 to 11 show the progress of the solution with the number of iterations. Similar results were found for temperature. In Figure 11 the $x$-velocity is also compared with the velocity profile from Ref. 18 .

\begin{tabular}{|c|c|c|}
\hline $\begin{array}{r}\text { Iteration } \\
(\times 1000)\end{array}$ & Unmodified & LTS \\
\hline 50 & $2 \mathrm{~h} 45 \min 46 \mathrm{~s}$ & $3 \mathrm{~h} 19 \mathrm{~min} 48 \mathrm{~s}$ \\
\hline 100 & $5 \mathrm{~h} 25 \mathrm{~min} 5 \mathrm{~s}$ & $6 \mathrm{~h} 30 \mathrm{~min} 32 \mathrm{~s}$ \\
\hline 150 & $8 \mathrm{~h}, 2 \mathrm{~min} 17 \mathrm{~s}$ & $9 \mathrm{~h} 41 \mathrm{~min} 21 \mathrm{~s}$ \\
\hline 300 & $15 \mathrm{~h} 49 \mathrm{~min} 2 \mathrm{~s}$ & - \\
\hline 600 & $31 \mathrm{~h} 12 \mathrm{~min} 9 \mathrm{~s}$ & - \\
\hline 900 & $46 \mathrm{~h} 23 \mathrm{~min} 12 \mathrm{~s}$ & - \\
\hline 1200 & $61 \mathrm{~h} 31 \mathrm{~min} 8 \mathrm{~s}$ & - \\
\hline 1500 & $76 \mathrm{~h} 42 \mathrm{~min} 21 \mathrm{~s}$ & - \\
\hline 1700 & $86 \mathrm{~h} 48 \mathrm{~min} 56 \mathrm{~s}$ & - \\
\hline
\end{tabular}

Table 3. Computational time required to reach each iteration level, for the unmodified and LTS solvers

\begin{tabular}{|c|c|c|}
\hline - - It $300 \mathrm{k}$ & $--\quad$ It $900 \mathrm{k}$ & - It $1500 \mathrm{k}$ \\
\hline - - It $600 \mathrm{k}$ & It $1200 \mathrm{k}$ & It $1700 \mathrm{k}$ \\
\hline
\end{tabular}

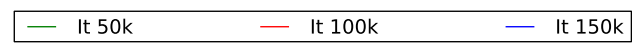

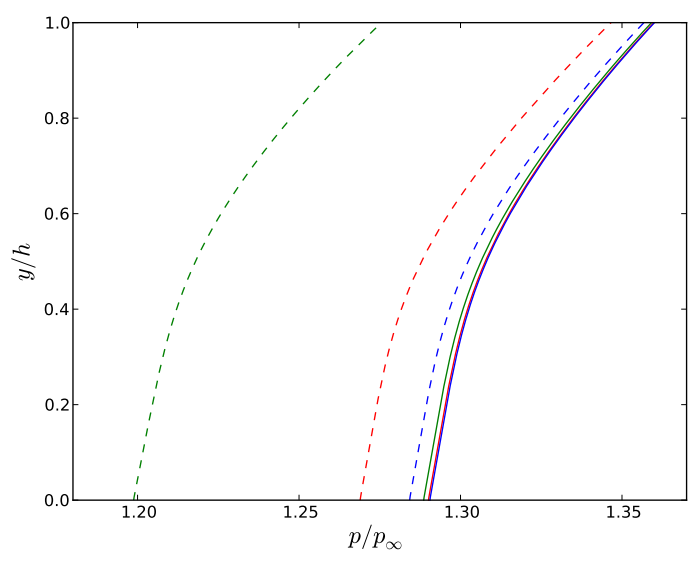

(a) Unmodified solver: $\mathrm{p}$

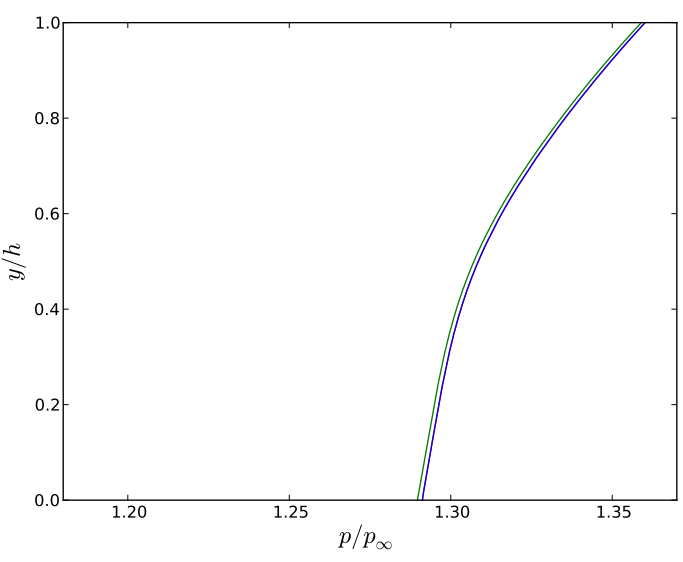

(b) LTS solver: p

Figure 9. Results from the unmodified and the LTS solvers for pressure 


\begin{tabular}{|c|c|c|}
\hline-- It $300 k$ & -- It $900 \mathrm{k}$ & - It 1500k \\
\hline-- It $600 \mathrm{k}$ & It $1200 \mathrm{k}$ & - It $1700 \mathrm{k}$ \\
\hline
\end{tabular}

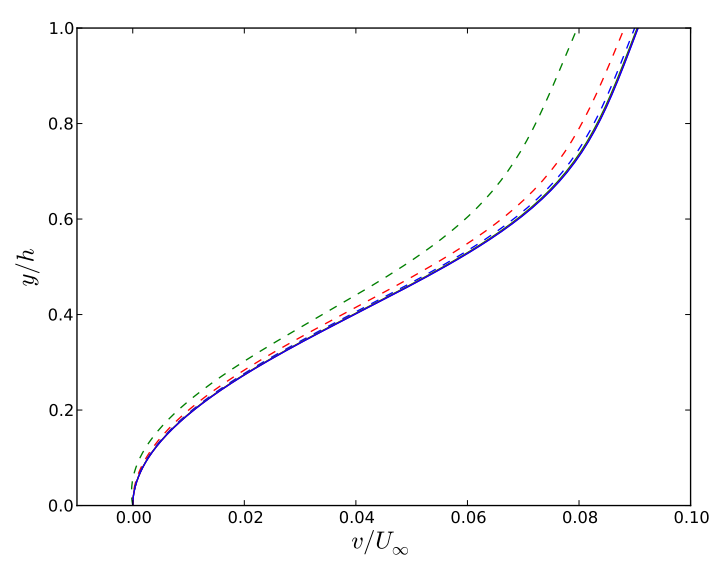

(a) Unmodified solver: $v$
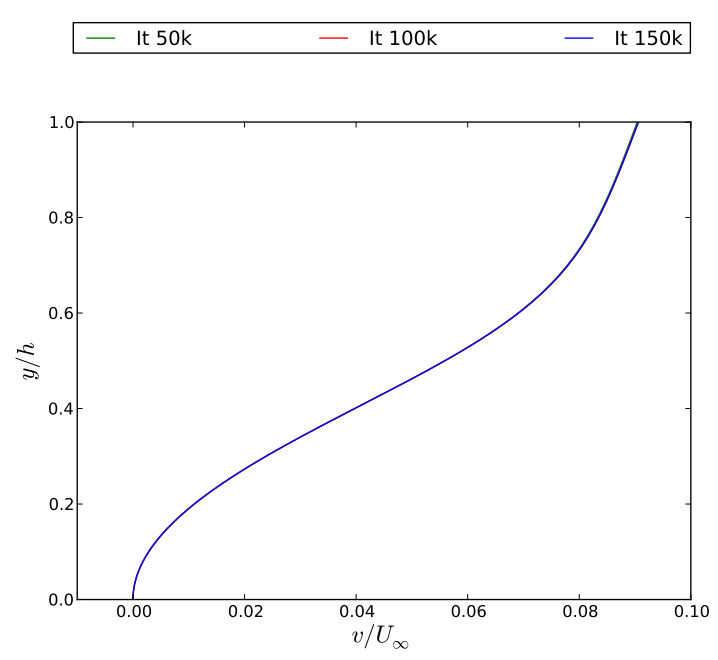

(b) LTS solver: v

Figure 10. Results from the unmodified and the LTS solvers for $y$-velocity

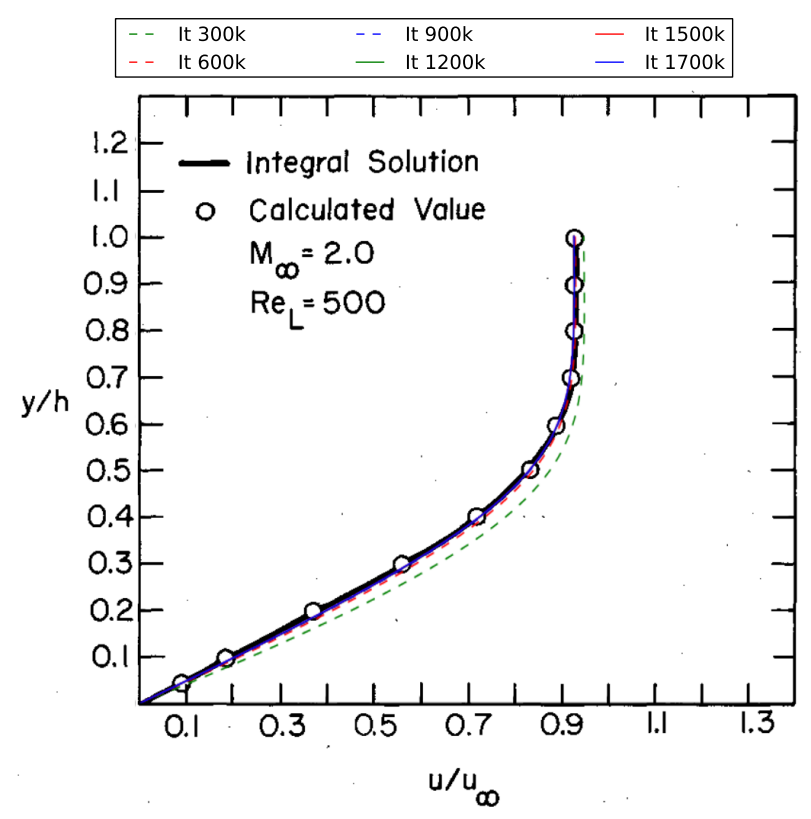

(a) Unmodified solver: $u$

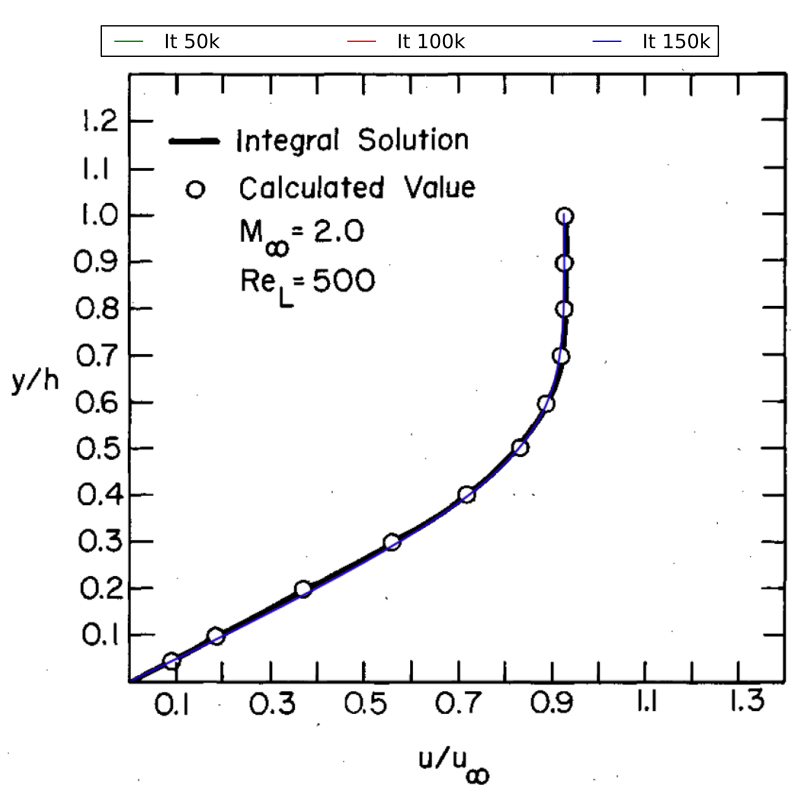

(b) LTS solver: u

Figure 11. Numerical laminar boundary layer profiles ( $x$-velocity) from Ref. 18 and present work, for the unmodified and LTS solvers 


\section{III.B. AMR}

\section{III.B.1. Supersonic wedge}

Figure 12 shows Mach number contour plots for the initial and refined meshes, as well as the meshes themselves. The shock wave is resolved with higher accuracy as the cell resolution is increased. This increase in accuracy can be also observed in Figure 13, where the analytical shock wave (black line) is superimposed on the previous Mach contour plots.

In Figure 14, the results for pressure, temperature, and Mach number on the top boundary are compared with the analytical solution, showing again the importance of cell refinement in the proximities of the shock wave.

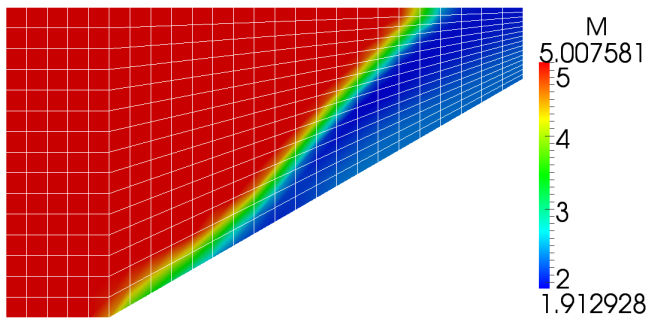

(a) Base mesh

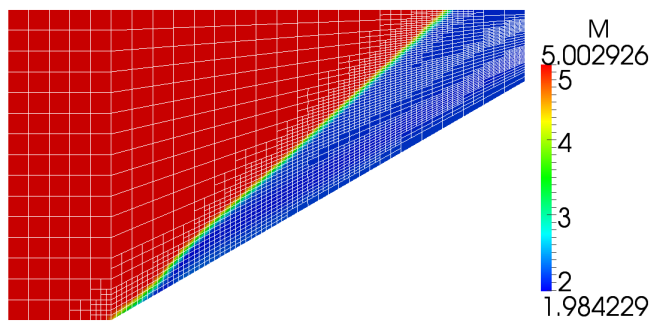

(c) Second Refinement

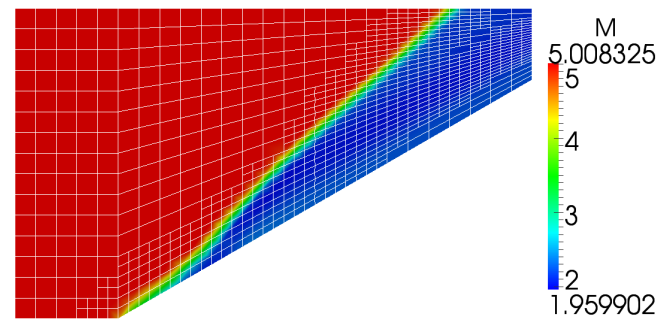

(b) First Refinement

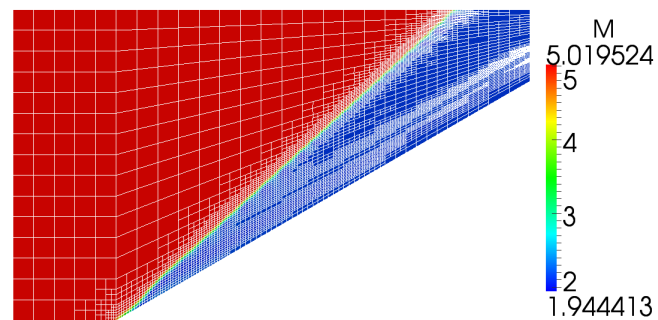

(d) Third Refinement

Figure 12. Mach number contour plots for different refinement levels

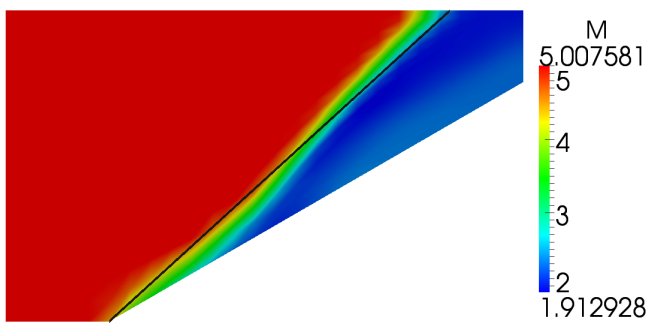

(a) Base mesh

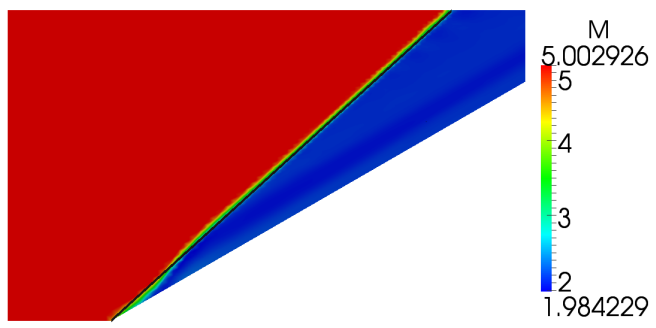

(c) Second Refinement

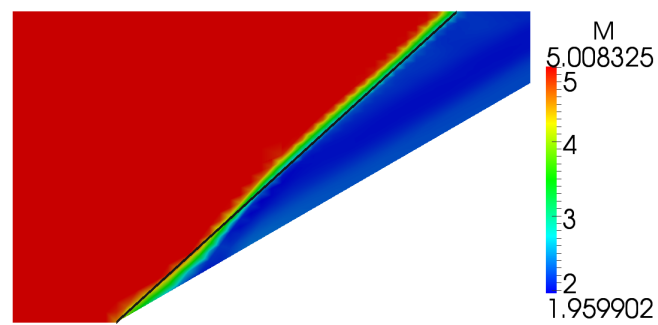

(b) First Refinement

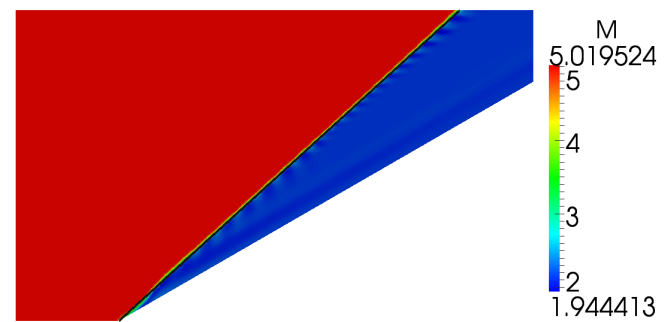

(d) Third Refinement

Figure 13. Comparison between Mach contour plots and analytical position of the shock wave for different refinement levels 


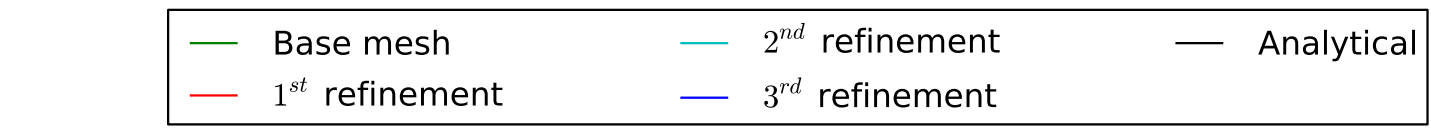

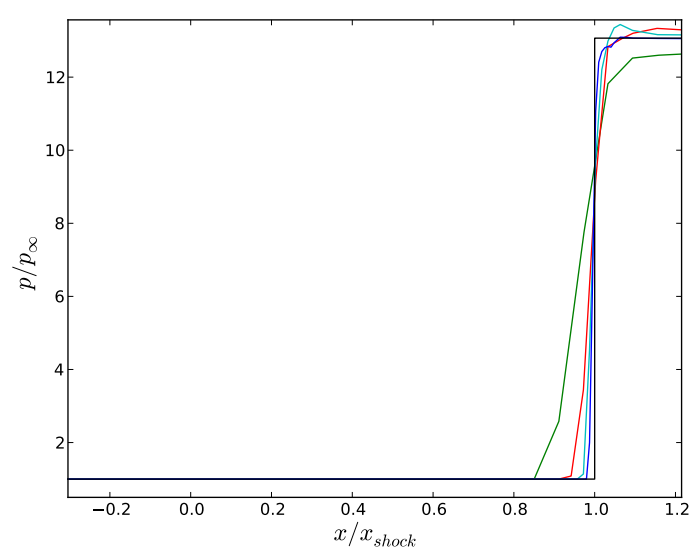

(a) Pressure

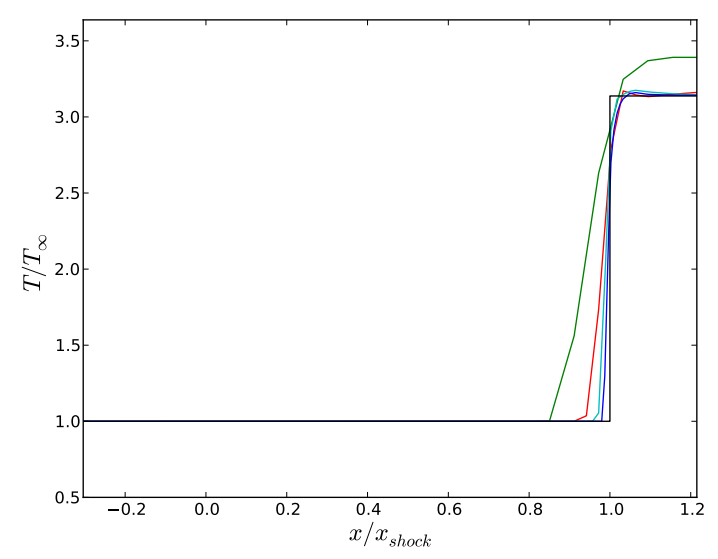

(b) Temperature

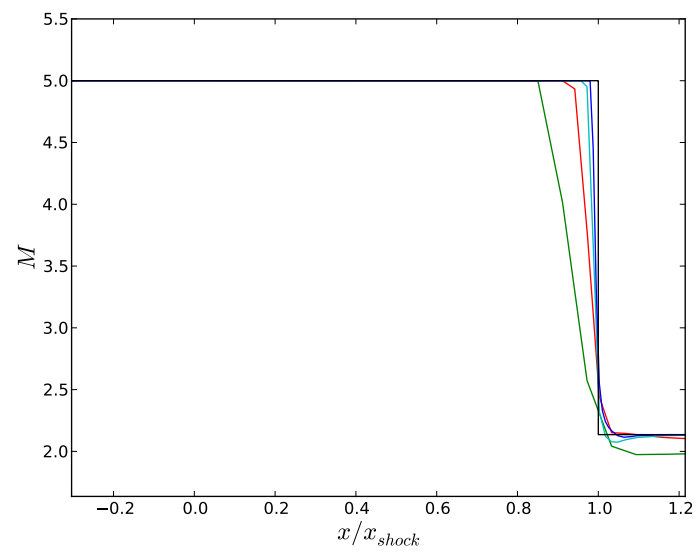

(c) Mach number

Figure 14. Solution comparison for the initial and refined meshes 


\section{III.B.2. Sod shock tube}

Figure 15 shows density contour plots for both refinement criteria, as well as the meshes themselves, for $\mathrm{t}=0.1 \mathrm{~s}$ and $\mathrm{t}=0.2 \mathrm{~s}$. The two refinement criteria were $\frac{\nabla p}{p}$ and $\frac{\nabla \rho}{\rho}$. Both the shock wave and the rarefaction wave are resolved with high accuracy, as the cell resolution is increased. However, it is evident that the $\frac{\nabla p}{p}$ criterion does not provide enough resolution to accurately capture the contact discontinuity. These features can also be observed in Figure 16, where the analytical solution for the shock tube is compared with the computational results.

The explanation for this lack of refinement near the contact discontinuity is that whereas density changes through this type of discontinuity, pressure does not. This makes the contact discontinuity invisible to the $\frac{\nabla p}{p}$ criterion, and reflects the importance of selecting an appropriate refinement criterion.

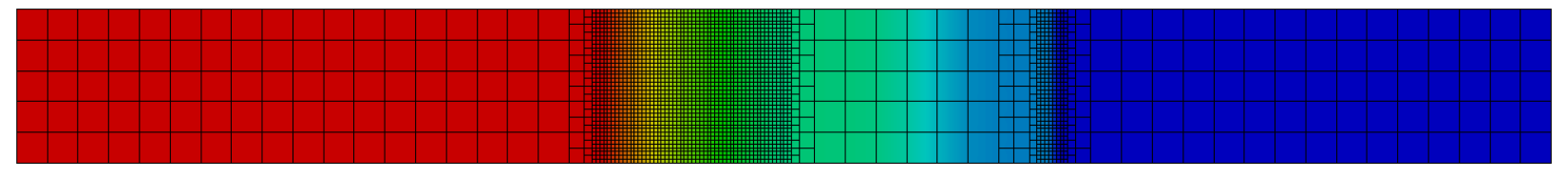

(a) $\nabla p / p$ criterion, $\mathrm{t}=0.1 \mathrm{~s}$

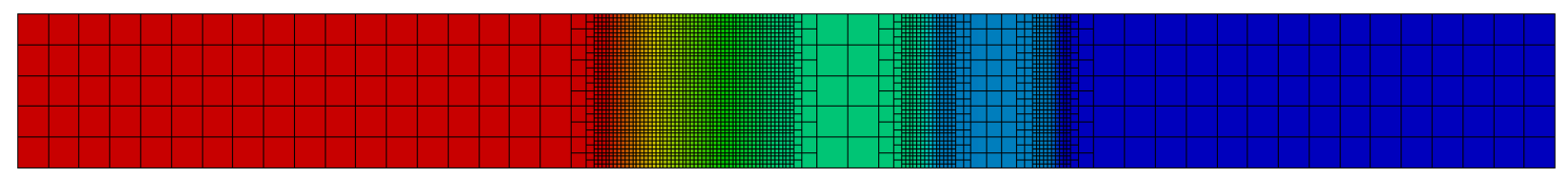

(b) $\nabla \rho / \rho$ criterion, $\mathrm{t}=0.1 \mathrm{~s}$

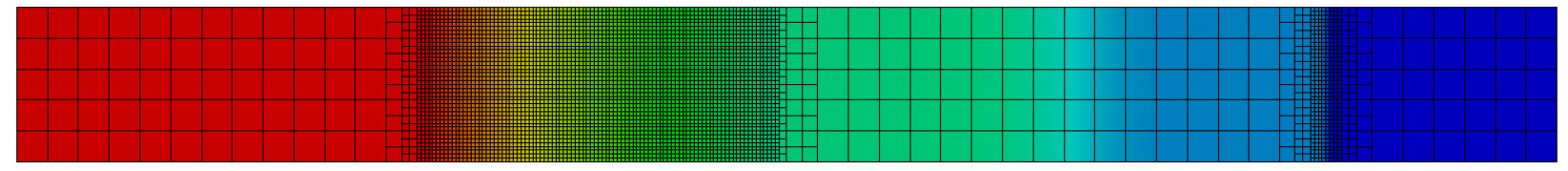

(c) $\nabla p / p$ criterion, $\mathrm{t}=0.2 \mathrm{~s}$

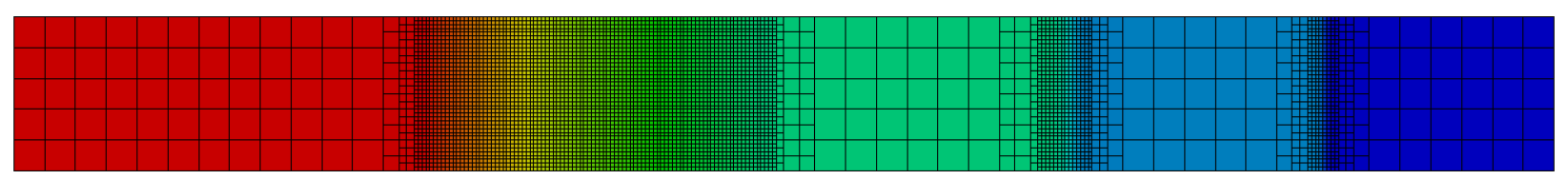

(d) $\nabla \rho / \rho$ criterion, $\mathrm{t}=0.2 \mathrm{~s}$

Figure 15. $\rho$ contour plots for the different refinement criteria, for $t=0.1 \mathrm{~s}$ and $\mathrm{t}=0.2 \mathrm{~s}$ 


\begin{tabular}{|c|c|}
\hline - Base mesh & $\operatorname{AMR}(\nabla \rho / \rho)$ \\
\hline AMR $(\nabla p / p)$ & Analytical \\
\hline
\end{tabular}

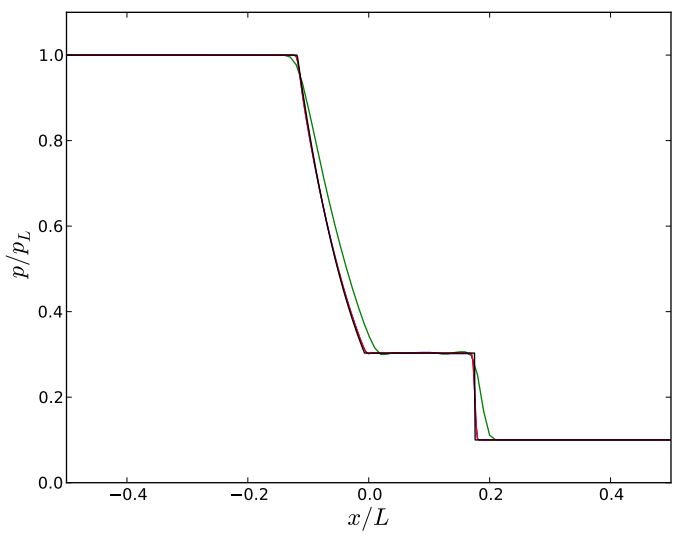

(a) Pressure, $t=0.1 \mathrm{~s}$

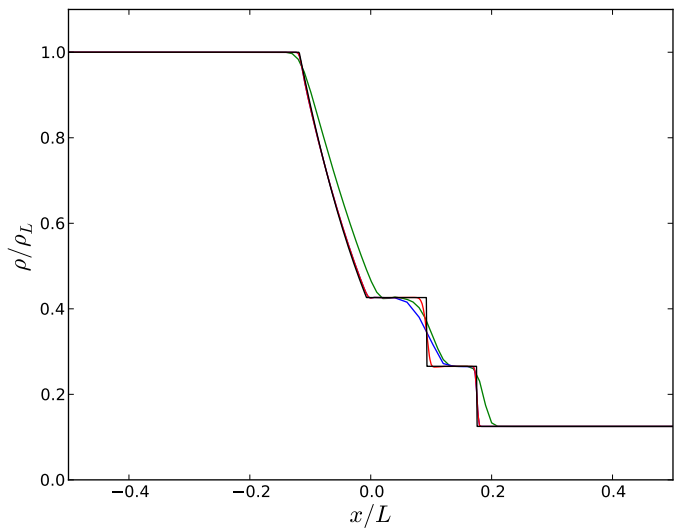

(c) Density, $\mathrm{t}=0.1 \mathrm{~s}$

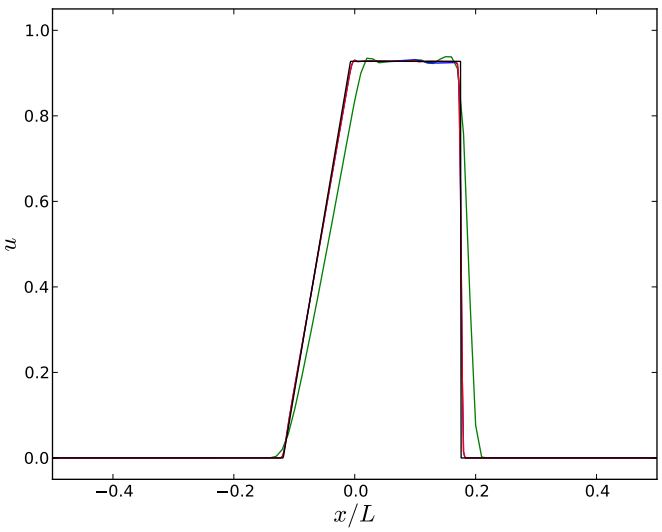

(e) Velocity, $\mathrm{t}=0.1 \mathrm{~s}$

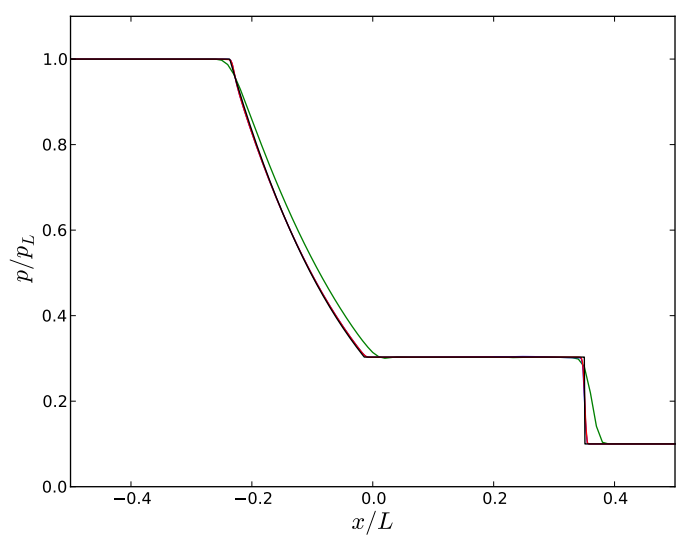

(b) Pressure, $\mathrm{t}=0.2 \mathrm{~s}$

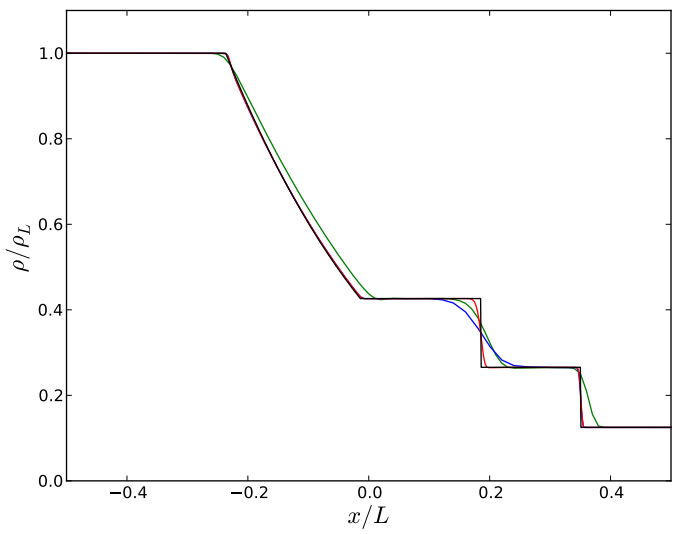

(d) Density, $\mathrm{t}=0.2 \mathrm{~s}$

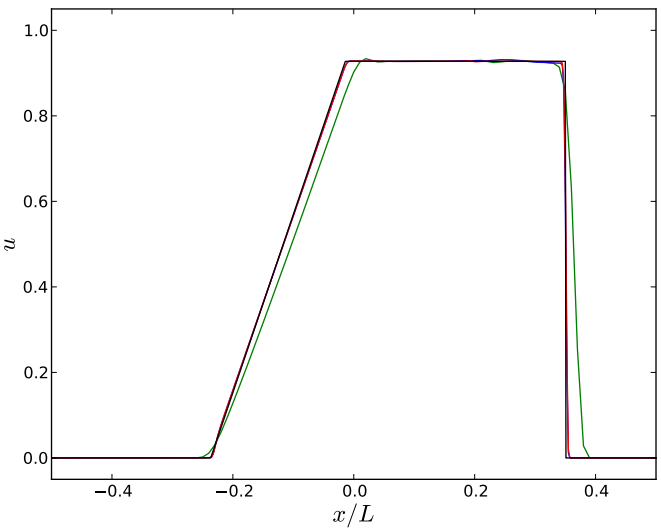

(f) Velocity, $\mathrm{t}=0.2 \mathrm{~s}$

Figure 16. Solution comparison for initial and refined meshes, for $t=0.1 \mathrm{~s}$ and $\mathrm{t}=0.2 \mathrm{~s}$ 


\section{Conclusions}

The local time stepping (LTS) and adaptive mesh refinement (AMR) techniques have been implemented into one of the OpenFOAM compressible flow solvers, rhoCentralFoam. The implementation of the LTS technique has resulted in a significant reduction of the computational time for the simulation of the Couette flow, matching the analytical solution to within $1.03 \%$ error 2.56 times faster than the unmodified solver. A more significant improvement was obtained for the flat plate, as the solution converged 8.96 times faster using local time stepping. The AMR technique has also been successfully implemented in rhoCentralFoam, producing an improved solution using significantly fewer computational cells compared with meshing procedures using global refinement. Future studies will apply the AMR and LTS techniques to additional high-speed compressible flow situations in order to fully categorise the extent of the computational time saving when applying these features.

\section{References}

\footnotetext{
${ }^{1}$ Maciel, E. and Pimenta, A., "Reentry Flows in Chemical Non-Equilibrium in Three-Dimensions," WSEAS Transactions on Mathematics, Vol. 11, No. 3, March 2012, pp. 262-282.

${ }^{2}$ Georgiadis, N., Yoder, D., Vyas, M., and Engblom, W., "Status of Turbulence Modeling for Hypersonic Propulsion Flowpaths," Theoretical and Computational Fluid Dynamics, Vol. 28, No. 3, June 2014, pp. 295-318.

${ }^{3}$ Bonfiglioli, A. and Paciorri, R., "Hypersonic Flow Computations on Unstructured Grids: Shock-Capturing vs. ShockFitting Approach," 40th Fluid Dynamics Conference and Exhibit, June 2010.

${ }^{4}$ Fico, V., Emerson, D., and Reese, J., "A Parallel Compact-TVD Method for Compressible Fluid Dynamics Employing Shared and Distributed-Memory Paradigms," Computers \& Fluids, Vol. 45, 2011, pp. 172-176.

${ }^{5}$ Wang, C., Wu, S. P., and Cao, N., "Application of Improved TVD Scheme in Hypersonic Heat-Flux Simulation," Advanced Materials Research, Vol. 588, 2012, pp. 1822-1826.

${ }^{6}$ Gnoffo, P. and White, J., "Computational Aerothermodynamic Simulation Issues on Unstructured Grids," 37th AIAA Thermophysics Conference, June 2004.

${ }^{7}$ Gnoffo, P. A., "Simulation of Stagnation Region Heating in Hypersonic Flow on TetrahedralGrids," 18 th AIAA CFD Conference, June 2007.

${ }^{8}$ Saunders, D. A., Yoon, S., and Michael, J. W., "An Approach to Shock Envelope Grid Tailoring and Its Effect on Reentry Vehicle Solutions," 45th AIAA Aerospace Sciences Meeting and Exhibit, Jan. 2007.

${ }^{9}$ Sha, P., Dinghua, P., Guohao, D., Zhengyu, T., Yueming, Y., and L., H., "Grid Dependency and Convergence of Hypersonic Aerothermal Simulation," Acta Aeronautica et Astronautica Sinica, Vol. 31, No. 3, March 2010.

${ }^{10}$ Gnoffo, P., "An Upwind-Biased, Point-Implicit Relaxation Algorithm for Viscous, Compressible Perfect-Gas Flows," Tech. rep., NASA Technical Report 2953, 1990.

${ }^{11}$ Wright, M., Candler, G., and Bose, D., "Data-Parallel Line Relaxation Method of the Navier-Stokes Equations," AIAA Journal, Vol. 36, No. 9, Sept. 1998.

12 www . openfoam. com, Accessed: 2014-10-23.

${ }^{13}$ Greenshields, C., Weller, H., Gasparini, L., and Reese, J., "Implementation of Semi-Discrete, Non-Staggered Central Schemes in a Colocated, Polyhedral, Finite Volume Framework, for High-Speed Viscous Flows," Journal for Numerical Methods in Fluids, Vol. 63, 2010, pp. 1-21.

${ }^{14}$ Gutiérrez, L. F., Tamagno, J. P., and Elaskar, S. A., "High Speed Flow Simulation Using OpenFOAM," Mecánica Computacional, Vol. XXXI, 2012, pp. 2939-2959.

${ }^{15}$ Kurganov, A. and Tadmor, E., "New High-Resolution Central Schemes for Nonlinear Conservation Laws and ConvectionDiffusion Equations," Journal of Computational Physics, Vol. 160, No. 1, 2000, pp. 241-282.

${ }^{16}$ Kurganov, A., Noelle, S., and Petrova, G., "Semi-Discrete Central-Upwind Schemes for Hyperbolic Conservation Laws and Hamilton-Jacobi Equations," SIAM J. Sci. Comput., Vol. 23, 2000, pp. 707-740.

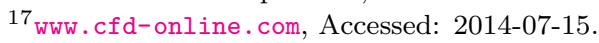

${ }^{18}$ Berman, H. A., Anderson, Jr., J. D., and Drummond, J. P., "Supersonic Flow over a Rearward Facing Step with Transverse Nonreacting Hydrogen Injection," AIAA Journal, Vol. 21, No. 12, 1983, pp. 1707-1713.

${ }^{19}$ Sod, G., "A Survey of Several Finite Difference Methods for Systems of Nonlinear Hyperbolic Conservation Laws," Journal of Computational Physics, Vol. 27, 1978, pp. 1-31.
} 